\title{
Inducible Rubicon facilitates viral replication by antagonizing interferon production
}

\author{
Yushun Wan ${ }^{1}$, Wei Cao ${ }^{1}$, Tao Han ${ }^{1}$, Sheng Ren ${ }^{1}$, Jian Feng ${ }^{1}$, TieLong Chen ${ }^{2}$, Jun Wang ${ }^{1}$, \\ Ruth Broering ${ }^{3}$, Mengji $\mathrm{Lu}^{4}$ and Ying $\mathrm{Zhu}^{1}$
}

The RUN domain Beclin-1-interacting cysteine-rich-containing (Rubicon) protein is involved in the maturation step of autophagy and the endocytic pathway as a Beclin-1-binding partner, but little is known regarding the role of Rubicon during viral infection. Here, we performed functional studies of the identified target in interferon (IFN) signaling pathways associated with Rubicon to elucidate the mechanisms of viral resistance to IFN. The Rubicon protein levels were elevated in peripheral blood mononuclear cells, sera and liver tissues from patients with hepatitis B virus (HBV) infection relative to those in healthy individuals. Assays of the overexpression and knockdown of Rubicon showed that Rubicon significantly promoted HBV replication. In addition, Rubicon knockdown resulted in the inhibition of enterovirus 71 , influenza $A$ virus and vesicular stomatitis virus. The expression o0f Rubicon led to the suppression of virus-induced type-I interferon (IFN- $\alpha$ and IFN- $\beta$ ) and type-III interferon (IFN- $\lambda 1$ ). Translocation of activated IRF3 and IRF7 from the cytoplasm to the nucleus was involved in this process, and the NF-кB essential modulator (NEMO), a key factor in the IFN pathway, was the target with which Rubicon interacted. Our results reveal a previously unrecognized function of Rubicon as a virus-induced protein that binds to NEMO, leading to the inhibition of type-I interferon production. Rubicon thus functions as an important negative regulator of the innate immune response, enhances viral replication and may play a role in viral immune evasion.

Cellular \& Molecular Immunology (2017) 14, 607-620; doi:10.1038/cmi.2017.1; published online 10 April 2017

Keywords: host response; immune evasion; interferon; Rubicon; viral infection

\section{INTRODUCTION}

Innate immunity is the first line of host defense against viral infection. According to their usage of three distinct receptors, ${ }^{1}$ IFNs are divided into at least three distinct types: types I, II and III. $^{2}$ Type-I IFNs, mainly IFN- $\alpha$ and IFN- $\beta$, play an important role in controlling viral replication during the initial stage of infection. $^{3}$ Type-I IFN $($ IFN- $\alpha / \beta)$ is a critical first line of defense that limits viral expression and replication., ${ }^{4,5}$ Recent advances have provided a clearer picture of the virus-triggered type-I IFN signaling pathways.

NF- $\kappa B$ essential modulator (NEMO, also known as IKK $\gamma$ ) ${ }^{6,7}$ has a central role in controlling many cellular processes in response to various physiological and pathological stimuli. NEMO is a poly-ubiquitin-binding protein and is essential for IFN- $\beta$ production, which is triggered by activated TANKbinding kinase 1 (TBK1) and IкB kinase epsilon (IKKe) as part of the antiviral process. ${ }^{8}$ Upon viral infection, NEMO interacts with TANK and forms the NEMO-TANK-TBK1 and IKKe complex, in which TBK1 and IKKe are activated by the phosphorylation of Ser172 in their activation loops. ${ }^{9}$ The activation of TBK1 and IKKe in turn activates IFN regulatory factor 3 (IRF3), a transcription factor required for IFN- $\beta$ gene transcription. ${ }^{10,11}$ The absence of NEMO impedes the IRF3 phosphorylation and type-I IFN production induced by Sendai virus infection. ${ }^{12}$

The RUN domain Beclin-1-interacting cysteine-rich-containing (Rubicon) protein was recently identified as a Beclin-1binding partner that localizes to the late endosome/lysosome and negatively regulates the maturation step of autophagy and the endocytic pathway. ${ }^{13,14}$ Under normal and stressful conditions, Rubicon primarily associates with the Beclin-1containing autophagy complex. Under Toll-like receptor (TLR)

\footnotetext{
${ }^{1}$ State Key Laboratory of Virology and College of Life Sciences, Wuhan University, Wuhan 430072, China; ${ }^{2}$ Department of Infectious Diseases, Zhongnan Hospital of Wuhan University, Wuhan 430072, China; ${ }^{3}$ Medical Faculty, Department of Gastroenterology and Hepatology, University of Duisburg-Essen, Essen 45127, Germany and ${ }^{4}$ Institute of Virology, University of Duisburg-Essen, Essen 45127, Germany Correspondence: Professor Y Zhu, PhD, State Key Laboratory of Virology and College of Life Sciences, Wuhan University, Wuhan 430072 , China. E-mail: yingzhu@whu.edu.cn

Received: 8 September 2016; Revised: 13 December 2016; Accepted: 13 December 2016
} 
activation, Rubicon is an essential positive regulator of the NADPH oxidase complex and a specific feedback inhibitor of CARD9-mediated PRR-signal transduction to prevent unbalanced proinflammatory responses. ${ }^{15}$ Thus, Rubicon regulates both autophagy and phagocytosis, depending on the environmental stimulus and is perfectly positioned to coordinate different but related innate immune mechanisms.

Although several functions of Rubicon have been described, a clear role of Rubicon in the innate immune response to viral infections has not been established. Human hepatitis B virus (HBV) has its ability to escape the host immune system. ${ }^{16-18}$ Here, we found that Rubicon can be induced by infection by viruses such as HBV, enterovirus 71 (EV71), influenza A virus (IAV) and vesicular stomatitis virus (VSV) and was a negative regulator for distributed IRF3 activation, IFN induction, and cellular antiviral response. Rubicon interacted with NEMO and suppressed TBK1 phosphorylation. Our findings provide novel insight to the molecular mechanisms underlying the IFNregulated antiviral response.

\section{MATERIALS AND METHODS}

Study subjects

Sixty-five patients with chronic hepatitis B and 65 healthy control individuals were recruited for the study between March 2014 and February 2015. The study was conducted according to the principles of the Declaration of Helsinki Principles and approved by the Institutional Review Board of the College of Life Sciences, Wuhan University, in accordance with institutional guidelines for the protection of human subjects. All participants provided written informed consent to participate in the study.

\section{Peripheral blood mononuclear cell and primary human hepatocyte isolation and transfection}

Peripheral blood mononuclear cells (PBMCs) were isolated by density centrifugation with an isolation solution of human lymphocytes (TBD Science) as previously described. ${ }^{19}$ The cells were washed twice in saline and cultured in RPMI 1640 medium. PBMCs were transfected with plasmid DNA by electroporation with an Amaxa Nucleofector II device according to the manufacturer's protocols.

Primary human hepatocytes (PHHs) were isolated from a single human liver specimen. Liver cells were prepared under a biosafety hood according to a modified two-step perfusion technique as previously described. ${ }^{20}$

\section{Reagents and constructs}

Puromycin (Invitrogen, Carlsbad, CA, USA); antibodies against IRF3, IRF7, IFN $\alpha$ PKR, OAS2, IKK $\alpha / \beta$, and TBK1 (Santa Cruz Biotechnology, Dallas, TX, USA); antibodies against Mxl, Rubicon, NEMO, and the protein of Rubicon (ProteinTech Group, Wuhan, China); antibodies against IFNAR1 (Abcam, Cambridge, MA, USA); antibodies against Flag, HA, Myc, and Rubicon (MBL, Woburn, Japan); and antibodies against phosphorylated TBK1 (Ser172), IRF3 (Ser396), and IKK $\alpha$ (Ser176)/IKK $\beta$ (Ser177; Cell Signal Tech, Beverly, MA, USA) were purchased from the indicated manufacturers. pHBV1.3 (genotype D, Serotype ayw, U95551), pHBV1.3 (genotype B, Serotype adw, JN406371), and IFN- $\lambda 1$ promoter were previously created in our laboratory. ${ }^{21,22}$ Flag-tagged NEMO and MAVS were created in our laboratory for this study. pHBV1.3 (genotype A, Serotype adw2, HE974381) was provided by Professor Xinwen Chen of Wuhan Institute of Virology, China. pHBV1.3 (genotype C, Serotype adr, FJ899793) was provided by Professor Dongping Xu of Beijing 302 Hospital, China. The $x$ gene mutant of HBV1.3 (pHBV1.3 $\Delta \mathrm{x})^{23}$ was a gift from Dan Liu of Zhongnan Hospital of Wuhan University, China. IFN- $\beta$ promoter and myc-Ubb were provided by Professor Hongbing Shu of Wuhan University, China. All plasmids that were received as gifts were confirmed by DNA sequencing. A fragment of the Rubicon promoter $(-1900$ to +100$)$ was amplified from human genomic DNA and subcloned into a pGL3-promoter vector (Promega, Madison, WI). The coding regions of Rubicon were purchased from Addgene (a gift from Qing Zhong (Addgene plasmid \# 28022) ${ }^{24}$ ) and generated by PCR amplification. The Rubicon amplicon was cloned into pCMV-Tag2B using EcoRI and XhoI and into pcAggs-HA using EcoRI and XhoI. The target of the Rubicon shRNAs was generated from a previously reported sequence and the Sigma-Aldrich website. To avoid off-target effects, we constructed two shRNAs with different target sites. The truncated Rubicon was amplified from those constructs and subcloned into the same empty vector according to previously described methods. $^{25}$

\section{Virus and cell culture}

The human hepatoma cell lines HepG2, Huh7 and A549 and RD cells were grown in DMEM (Gibco, Waltham, MA, USA) supplemented with $10 \%$ heat-inactivated fetal bovine serum (FBS; Gibco), $100 \mathrm{U} / \mathrm{ml}$ penicillin, and $100 \mathrm{U} / \mathrm{ml}$ streptomycin sulfate (Gibco) at $37^{\circ} \mathrm{C}$ in $5 \% \mathrm{CO}_{2}$. The HepG2.2.15 cell line stably expressing $\mathrm{HBV}$ was used as previously described. ${ }^{21}$ The knockdown lines of Huh7 (Scr-K.D. and Rub-K.D.) were cultured in DMEM with $10 \%$ FBS and $300 \mu \mathrm{g} / \mathrm{ml}$ puromycin (Gibco). ${ }^{26}$ The influenza virus strain A/Hong Kong/498/97 and the Indiana serotype of VSV were provided by the China Center Type Culture Collection. EV71 was provided by Renmin Hospital of Wuhan (Hubei, China). The HBV particles used for $\mathrm{PHH}$ infection were harvested from HepG2.117 cells. For HBV infection, PHH cells were cultured in primary hepatocyte maintenance medium (PMM) for $24 \mathrm{~h}$ and then inoculated with a 200 multiplicity of genome equivalents of $\mathrm{HBV}$ in PMM with $4 \%$ PEG 8000 at $37^{\circ} \mathrm{C}$ for $\sim 24 \mathrm{~h}$. One day after infection, the cell were washed with phosphate-buffered saline (PBS) three times to remove residual viral particles and maintained in the same medium containing $2 \%$ dimethylsulfoxide. The medium was refreshed every other day.

\section{Transfection and luciferase reporter-gene assays}

Cells were seeded in 24-well or 6-well dishes, depending on the experiment, and grown to confluence (reaching 
approximately $80-90 \%$ at the time of transfection). The cells were transfected using Lipofectamine 2000 transfection reagent (Invitrogen) according to the protocol provided by the manufacturer. The Renilla luciferase reporter vector pRL-TK was used as an internal control. Luciferase assays were performed with a dual-specific luciferase assay kit (Promega, Madison, USA). Firefly luciferase activities were normalized based on the Renilla luciferase activities. All reporter assays were repeated at least three times. The data shown are average values \pm s.d. from one representative experiment.

HBV DNA quantification and quantitative RT-PCR analysis The purification and quantification of HBV DNA has been described in a previous study. ${ }^{27}$

Total RNA was isolated using TRIzol reagent (Invitrogen), treated with DNase I, and reverse-transcribed with MLV reverse transcriptase (Promega, Madison, WI, USA) and random primers (Takara Bio, Kusatsu, Japan). Quantitative reverse transcriptasePCR (RT-PCR) was performed using a LightCycler 480 (Roche, Basel, Switzerland) and the SYBR Green system (Roche, Basel, Switzerland). GAPDH or actin was amplified as an internal control. The primers used are listed below:

GAPDH forward-5'-GGAAGGTGAAGGTCGGAGTCA ACGG-3' reverse-5'-CTCGCTCCTGGAAGATGGTGATGGG-3' ; PKR forward-5'-AAAGCGAACAAGGAGTAAG-3' reverse5'-GATGATGCCATCCCGTAG-3'; OAS forward-5'-TTCCG TCCATAGGAGCCAC-3' reverse-5'-AAGCCCTACGAAGAAT GTC-3'; MxA forward-5'-GCCGGCTGTGGATATGCTA-3' reverse-5'-TTTATCGAAACATCTGTGAAAGCAA-3'; Rubicon forward-5'-AACCTCACCCACCATCTTCTTAGCGT-3' reverse$5^{\prime}$-CACAGAGTTAAGTGCATAATTGGCATAAAGG-3'; IFN- $\beta$ forward-5'-TGGGAGGCTTGAATACTGCCTCAA- $3^{\prime}$ reverse$5^{\prime}$-TCCTTGGCCTTCAGGTAATGCAGA- $3^{\prime} ;$ IFN- $\alpha$ forward$5^{\prime}$-TTTCTCCTGCCTGAAGGACAG-3' reverse- $5^{\prime}$-GCTCATG ATTTCTGCTCTGACA- $3^{\prime} ; \quad$ IFN- $\lambda 1$ forward-5'-GTGACTTT GGTGCTAGGCTTG-3' reverse-5'-GCCTCAGGTCCCAATT CCC-3'; NP (IAV) forward-5'-ATCAGACCGAACGAGAATC CAGC-3' reverse-5'-GGAGGCCCTCTGTTGATTAGTGT-3'; VP1 (EV71) forward-5'-AATTGAGTTCCATAGGTG-3' reverse5'-CTGTGCGAATTAAGGACAG-3'.

\section{Northern blot analysis}

Whole-cell lysates were prepared using TRIzol. RNA was extracted, and Northern blot was performed using the DIG high-prime DNA labeling and detection starter kit II (Roche, Basel, Switzerland).

\section{Western blot analysis}

Whole-cell lysates were prepared by lysing cells with PBS (pH 7.4) containing $0.01 \%$ Triton X-100, 0.01\% EDTA, and $10 \%$ protease inhibitor cocktail (Roche). The protein concentration was determined by the Bradford assay (Bio-Rad, CA, USA). The polypeptides from cell lysates were separated on SDS 12\% polyacrylamide gels, crosslinked with $\mathrm{N}, \mathrm{N}$-methylenebisacylamide and transferred electrically to nitrocellulose membranes. Nonspecific binding was blocked with 5\% milk in PBST before addition of primary antibodies. Horseradish peroxidase-linked anti-rabbit, anti-mouse, or anti-goat antibodies (Santa Cruz Biotechnology) were used as secondary antibodies. Blots were developed using SuperSignal Chemiluminescent reagent (Pierce, Rockford, IL, USA), and the stained membranes were analyzed with a LAS-4000 image document instrument (Fujifilm, Tokyo, Japan).

\section{ELISA detection}

Serum Rubicon was quantified by ELISA. Recombinant Rubicon protein was purchased from Proteintech. ELISAs were performed by coating the bottom of a 96-well plate with clinical serum samples overnight at $4{ }^{\circ} \mathrm{C}$. The capture antibody was incubated for $2 \mathrm{~h}$ at room temperature, and then the plate was washed three times with TBST, followed by incubation with the appropriate HRP-labeled secondary antibody for $1 \mathrm{~h}$ and finally washing five times with TBST. ELISAs were developed using the substrate 3,3',5,5'-tetramethylbenzidine (Sigma-Aldrich, St Louis, MO, USA), and the absorbance at the double wavelength $450 / 630 \mathrm{~nm}$ was measured. Rubicon concentrations were calculated from the standard curve.

IFN-alpha in the supernatant was detected using the human IFN $\alpha$ kit purchased from R\&D Systems (Minneapolis, MN, USA).

\section{Nuclear extraction}

Cells were incubated in serum-free media for $24 \mathrm{~h}$, washed twice with cold PBS, and scraped into $1 \mathrm{ml}$ cold PBS, followed by centrifugation at 2000 r.p.m. for $10 \mathrm{~min}$ in a microcentrifuge and incubation in two packed cell volumes of buffer A (10 mM HEPES ( $\mathrm{pH}$ 8), 0.5\% NP-40, $1.5 \mathrm{mM} \mathrm{MgCl}_{2}$, $10 \mathrm{mM} \mathrm{KCl}, 0.5 \mathrm{mM}$ DTT and $200 \mathrm{mM}$ sucrose) for $10 \mathrm{~min}$ on ice with inversion of the tube to mix. Nuclei were then collected by centrifugation (12 000 r.p.m. for 15 s). The pellets were rinsed with buffer A, resuspended in buffer B $(20 \mathrm{mM}$ HEPES ( $\mathrm{pH} 7.9$ ), $1.5 \mathrm{mM} \mathrm{MgCl}, 420 \mathrm{mM} \mathrm{NaCl}, 0.2 \mathrm{mM}$ EDTA, and $1.0 \mathrm{mM} \mathrm{DTT}$ ), and incubated on a rocking platform for $30 \mathrm{~min}\left(4^{\circ} \mathrm{C}\right)$. Nuclei were clarified by centrifugation (12000 r.p.m. for $15 \mathrm{~min}$ ), and the supernatants were diluted 1:1 with buffer C (20 mM HEPES ( $\mathrm{pH} 7.9), 100 \mathrm{mM}$ $\mathrm{KCl}, 0.2 \mathrm{mM}$ EDTA, 20\% glycerol, and $1 \mathrm{mM}$ DTT). Cocktail protease inhibitors were added to each type of buffer. The nuclear extracts were stored at $-70^{\circ} \mathrm{C}$ until use.

\section{Immunofluorescence}

After transfection, the cells were fixed with $4 \%$ paraformaldehyde for $15 \mathrm{~min}$, washed three times with PBS, and permeabilized with PBS containing $0.5 \%$ Triton X-100 for 5 min. The cells were then washed three times with PBS and blocked with PBS containing 4\% BSA for $1 \mathrm{~h}$ at room temperature. The cells were then incubated with the primary antibody overnight at $4{ }^{\circ} \mathrm{C}$, followed by incubation with secondary antibodies (ProteinTech Group) for $1 \mathrm{~h}$. Mounting was performed with Vectashield mounting medium with DAPI (Vector Laboratories, Burlingame, CA, USA), and the cells were visualized by confocal 
laser microscopy (FLUOVIEW FV1000; Olympus, Tokyo, Japan).

\section{RNA interference}

Rubicon shRNA (shR) and control shRNA (Scr) was constructed or purchased from Sigma and prepared by ligation of the corresponding pairs of oligonucleotides to plKO.1. To avoid off-target effects, two shRNA expression plasmids for Rubicon were used. For the re-expression of Rubicon after knockdown, one of the shRNAs targeted the $3^{\prime}$ untranslated region ( $3^{\prime}$-UTR) of Rubicon. The target of the shRNA was (1) 5'-AAGATCGATGCGTCCATGTTT-3' and (2) 5'-CCTG CATGTCAGGTATCATTT-3'.

\section{Histology and immunohistochemistry}

Liver tissue was fixed in $4 \%$ formaldehyde and embedded in paraffin. Immunohistochemical staining was performed using a kit (EliVisionTMplus, Maixin, Inc., Fuzhou, China) according to the protocol provided by the manufacturer. ${ }^{22}$ Briefly, liver biopsy slides were heated at $60^{\circ} \mathrm{C}$ for $1 \mathrm{~h}$ and then deparaffinized and dehydrated. After antigen retrieval, the slides were subjected to a serum-free protein block, a peroxidase block, and an avidin-biotin system block before incubation with Rubicon antibody (Proteintech, 1:50 dilution) overnight at $4{ }^{\circ} \mathrm{C}$ in a humidified chamber. A universal secondary antibody was applied to each slide after several washes with buffer. The Rubicon antibody was visualized using the HRP DAB detection kit according to the protocol provided by the manufacturer. The slides were counterstained with hematoxylin, dehydrated, cleared with xylene and sealed with a coverslip using a mounting medium (Maixin, Inc.). Images were acquired by fluorescence microscopy (FLUOVIEW FV1000; Olympus, Tokyo, Japan).

\section{Co-immunoprecipitation}

Co-immunoprecipitation analysis was performed as previously described. ${ }^{28}$ Briefly, after cell treatment, the cells were collected and lysed using lysis buffer (20 mM Tris ( $\mathrm{pH} 7.5), 150 \mathrm{mM}$ $\mathrm{NaCl}, 0.5 \%$ (vol/vol) Nonidet-P40, $1 \mathrm{mM}$ EDTA, $30 \mathrm{mM} \mathrm{NaF}$, $2 \mathrm{mM}$ sodium pyrophosphate, $10 \mathrm{mg} / \mathrm{ml}$ aprotinin, and $10 \mathrm{mg} /$ $\mathrm{ml}$ leupeptin). The lysates were mixed and precipitated overnight at $4{ }^{\circ} \mathrm{C}$ with antibodies or IgG and protein G-agarose beads. The beads were washed five times with lysis buffer, and bound proteins were separated by SDS-PAGE with subsequent immunoblotting analysis.

\section{Statistical analysis}

All experiments were repeated at least three times. Statistical analyses were performed using paired Student's $t$-tests or one-way analysis of variance for multiple comparisons. A $P$-value $<0.05$ was considered statistically significant.

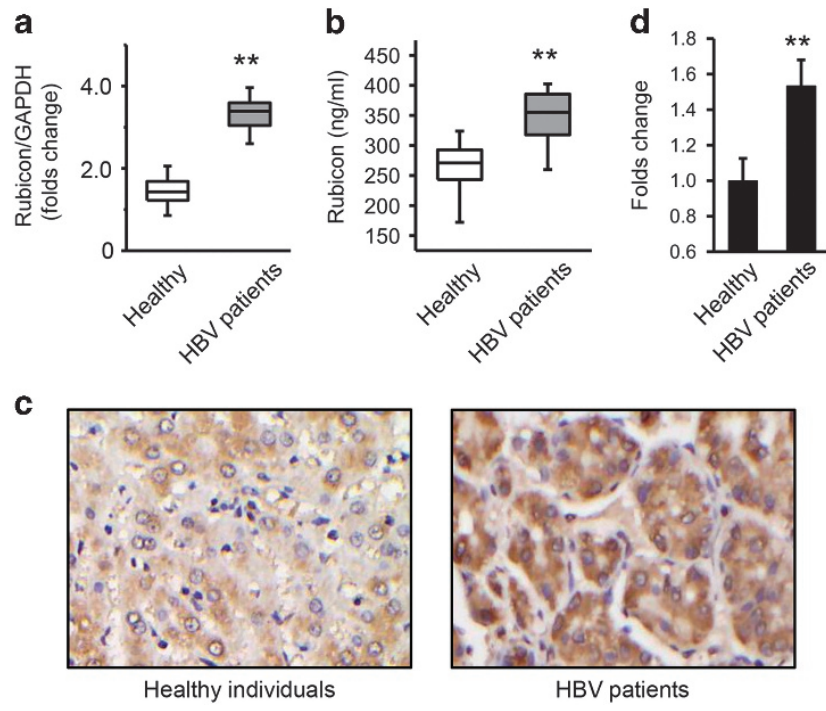

Figure 1 Rubicon expression in healthy individuals and in patients with HBV infection. (a) PBMCs of healthy individuals $(n=20)$ and patients with HBV infection $(n=20)$ were isolated for total RNA extraction. Rubicon mRNA was detected by qRT-PCR. (b) Rubicon levels were detected by ELISA in the sera of healthy individuals $(n=30)$ and patients with HBV infection $(n=30)$. (c) Immunohistochemical staining of Rubicon in normal livers $(n=15)$ and livers of patients with HBV infection $(n=15)$. (d) Quantitation of the immunohistochemical staining by ImageJ. All experiments were repeated at least three times with similar results. The data represent the mean \pm s.e., $n=3$. Bar graphs represent the mean \pm s.e., $n=3, \quad\left({ }^{*} P<0.01 ;{ }^{*} P<0.05\right) . \quad$ HBV, hepatitis B virus; PBMC, peripheral blood mononuclear cell; qRT-PCR, quantitative reverse transcriptase-PCR.

\section{RESULTS}

Rubicon expression is elevated in patients with chronic hepatitis $\mathrm{B}$

Although HCV is known to induce Rubicon expression in tissue culture, ${ }^{29}$ there have been no reports of Rubicon expression in patients infected with $\mathrm{HBV}$ or other viruses. We first investigated Rubicon expression during HBV infection. Peripheral blood mononuclear cells (PBMCs) were isolated from 20 patients with chronic hepatitis $\mathrm{B}(\mathrm{CHB})$ and from 20 healthy individuals. Real-time RT-PCR showed that Rubicon mRNA levels were $~ 2.18$-fold higher in PBMCs from the patients with $\mathrm{CHB}$ than in those from the healthy individuals (Figure 1a, Supplementary Table 1). ELISA showed similar results for serum Rubicon levels between 30 patients with $\mathrm{CHB}$ and 30 healthy individuals $(343.59 \pm 40.95$ versus $260.02 \pm 40.26 \mathrm{ng} / \mathrm{ml}$; Figure $1 \mathrm{~b}$ and Supplementary Table 2).

Immunohistochemistry showed higher levels of Rubicon staining in liver biopsy specimens from 15 patients with $\mathrm{CHB}$ compared with those in specimens from 15 healthy individuals (Figure 1c). We then used ImageJ software to quantitate the staining (Figure 1d). The data demonstrated that the expression of Rubicon was indeed higher in the HBVinfected biopsies.

There was a linear positive correlation between HBV DNA and the Rubicon mRNA levels in PBMCs $(n=20)$ and protein 
a
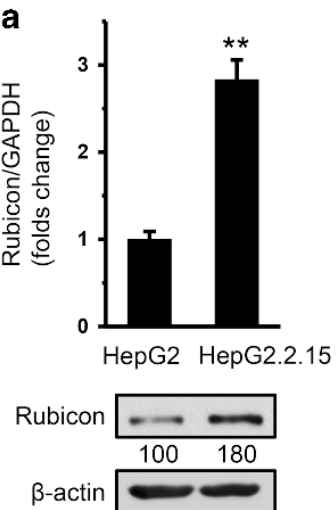

d

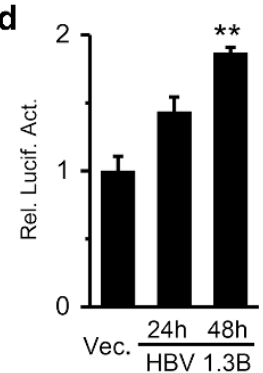

b
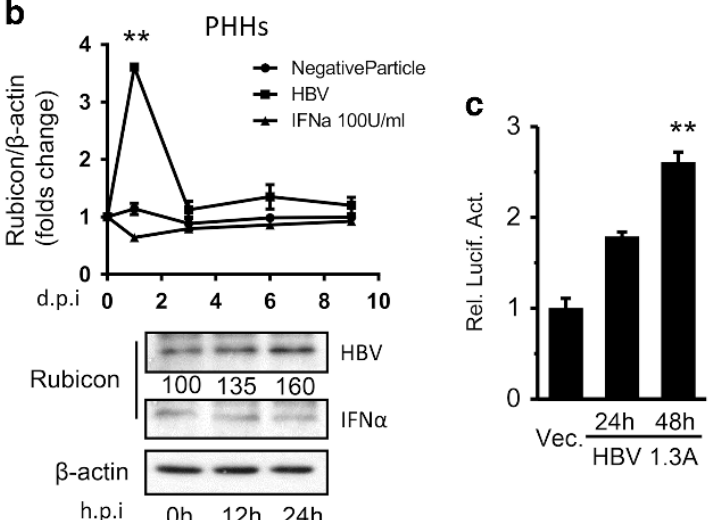

e

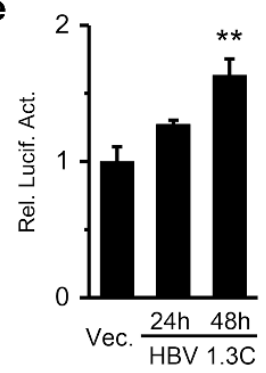

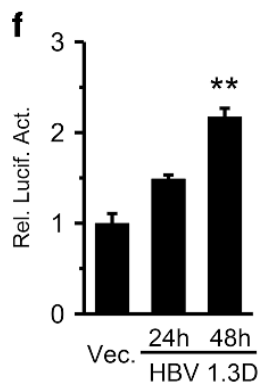

g

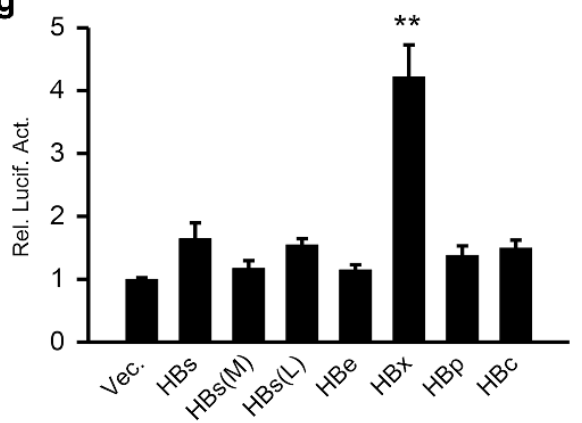

h

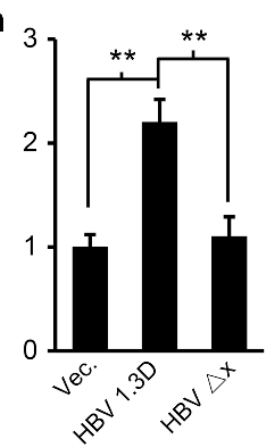

Figure 2 Rubicon expression induced by virus infection. (a) HepG2 and HepG2.2.15 cells were used to determine Rubicon expression by qRT-PCR (up) and western blotting (down). (b) Isolated PHHs from liver biopsies were cultured with mock or HBV particles or IFN- $\alpha$ $(100 \mathrm{U} / \mathrm{ml})$ for $24 \mathrm{~h}$. The cells were then collected to measure Rubicon expression by qRT-PCR (up) or western blotting (down) at the indicated times after infection. (c-f) Huh7 cells were co-transfected with luciferase reporter plasmids containing the Rubicon promoter (pRubicon-luc) and pHBV1.3A/pHBV1.3B/pHBV1.3C/ pHBV1.3D or vector. (g) Huh7 cells were co-transfected with pRubicon-luc and the indicated overexpression plasmid containing the HBV gene or vector. (h) Huh7 cells were co-transfected with pRubicon-luc and pHBV1.3D or pHBV1.3Ax or vector. The cell culture medium was refreshed with serum-free medium $12 \mathrm{~h}$ post transfection. Luciferase activities were measured after $24 \mathrm{~h}$ of serum starvation. All experiments were repeated at least three times with similar results. The data represent the mean \pm s.d., $n=3$. Bar graphs represent the mean \pm s.d., $n=3,\left({ }^{*} P<0.01 ;{ }^{*} P<0.05\right)$. IFN- $\alpha$, interferon- $\alpha$; HBV, hepatitis $B$ virus; qRT-PCR, quantitative reverse transcriptase-PCR.

levels in sera $(n=30)$ from patients with CHB (Supplementary Figures $1 \mathrm{~A}$ and $\mathrm{B}$ ); however, there were no significant differences in Rubicon expression based on sex, age or HBV genotype (data not shown).

\section{HBV enhances Rubicon expression in hepatocytes}

We assessed whether HBV infection could induce endogenous Rubicon in cell cultures. We measured Rubicon mRNA and protein levels in HepG2 human hepatoma cells and in HepG2.2.15 cells, which contain an integrated HBV (subtype ayw) genome and stably express HBV. The Rubicon mRNA and protein levels in the HepG2.2.15 cells were significantly higher than those in HepG2 cells (Figure 2a).

We transfected Huh7 cells with empty vector or with pHBV1.3, a plasmid containing a 1.3-fold over length fragment of the HBV genome (subtype ayw) that retains the ability to produce mature $\mathrm{HBV}$ virions. The $\mathrm{HBV}$ elevated the expression levels of Rubicon mRNA in the transfected cells (Supplementary Figure 2A).

HBV infects hepatocytes but it still can be detected in PBMCs at all stages during the infection cycle. ${ }^{21,22}$ We therefore tested the influence of HBV infection on Rubicon expression in PBMCs. 
We cultured freshly isolated PBMCs with the supernatants from HepG2 cells, HepG2.2.15 cells (containing $10^{5} \mathrm{HBV}$ virions/ml) or HepG2.2.15 cells pre-incubated with HBV immunoglobulins (anti-HBs). The Rubicon mRNA and protein levels in the PBMCs were increased by the HepG2.2.15 supernatant and decreased by the anti-HBs supernatant (Supplementary Figure 2B). These data suggest that HBV can induce Rubicon expression and that anti-HBs can inhibit the induction.

We also isolated $\mathrm{PHH}$ for $\mathrm{HBV}$ infection. To test whether the higher expression of Rubicon is a consequence of the IFN induction, we used IFN- $\alpha$ to treat PHHs and test the expression of Rubicon by qRT-PCR and western blotting at the indicated times (Figure $2 b$ ). The data showed that HBV infection could induce Rubicon expression at the early stage of viral infection, and the expression level dropped to normal levels after 3 days. In contrast, the treatment of IFN- $\alpha$ did not affect the expression of Rubicon at all times, indicating that high expression levels of Rubicon result from HBV infection rather than IFN.

We generated the Rubicon promoter from the human genomic DNA and inserted it into pGL3-basic to form pRubicon-luc. To determine the level at which HBV influences Rubicon expression, we co-transfected Huh7 cells with pRubicon-luc and pHBV1.3 (genotype A, B, C or D). Luciferase reporter assays revealed that all HBV subtypes activated the Rubicon promoter-luc in Huh7 cells (Figures 2c-f). All these subtypes activated the Rubicon promoter activities approximately twofold at $48 \mathrm{~h}$ after transfection.

HBV expresses seven proteins, ${ }^{30,31}$ and the $\mathrm{x}$ protein interferes with cellular processes and host transcription. ${ }^{32,33}$ Interestingly, only HBV $\mathrm{x}$ protein could induce Rubicon expression (Figure 2g) after pRubicon-luc and each $H B V$ gene had been co-transfected in Huh7 cells. Then, the $x$ gene early stopped $\mathrm{pHBV} 1.3 \Delta \mathrm{x}$ plasmid was used to verify these results. The luciferase activities of pRubicon-luc showed that the induction factor of Rubicon was $H B x$ (Figure $2 \mathrm{~h}$ ) but not the other gene.

All of the data suggest that HBV x protein induces Rubicon transcription and that the differences among HBV subtypes are small.

\section{Rubicon facilitates $\mathrm{HBV}$ replication}

HBV infection can induce Rubicon expression; next, we expected to explore the function of Rubicon during HBV replication. Huh7 cells were co-transfected with Rubicon expression plasmid and pHBV1.3 (genotype D). We then determined HBV replication by measuring viral protein expression and DNA copy numbers. The results showed that Rubicon overexpression stimulated the expression of $\mathrm{HBV} \mathrm{E}$ and $\mathrm{S}$ antigens (HBeAg and HBsAg; Figures $3 \mathrm{~A}$ and $\mathrm{B}$ ) and enhanced the replication of HBV DNA (Figure 3C). Overexpression of Rubicon in Huh7 cells was measured by western blotting (Figure 3D). In contrast, Rubicon knockdown by shRNA ( $\operatorname{sh} \# 1$ ) suppressed the levels of HBeAg (Figure 3E), HBsAg (Figure 3F), and HBV DNA (Figure 3G). To avoid off- target effects, we assessed the ability of another Rubiconspecific shRNA (shR\#2) to inhibit HBV replication and expression and observed similar results. We also tested the interference efficiency of the shRNAs in Huh7 cells (Figure $3 \mathrm{H}$ ).

We then measured the HBV RNA levels by northern blot and IFN $\alpha$, FNAR1 protein levels in enriched supernatant or cells by western blot. Rubicon overexpression enhanced HBV RNA expression, while the production of IFN $\alpha$ decreased (Figure 3I(a)). In contrast, stable knockdown of Rubicon in Huh7 cells by shR\#2, which targets the 3 '-UTR of the Rubicon mRNA, reduced the HBV RNA level significantly with upregulated IFN $\alpha$ production, while overexpression of Rubicon in the stable knockdown cells reversed this effect (Figure 3I(b)). In addition, the IFN $\alpha$ protein levels were also measured by ELISA (Figure 3J). All these data suggest that Rubicon can enhance HBV replication.

\section{EV71, IAV and VSV induce Rubicon expression, and Rubicon promotes virus replication and production}

To determine whether other viruses induce Rubicon expression, we infected RD, A549, and Huh7 cells with EV71 (Figure 4A), IAV (Figure 4B) and VSV (Figure 4C), respectively. Real-time RT-PCR and western blotting results indicated that all three viruses induced Rubicon mRNA and protein expression.

We measured the effect of Rubicon on EV71 replication by detecting viral VP1 mRNA in RD cells. Rubicon overexpression significantly enhanced the expression of VP1 (Figure $4 \mathrm{D}(\mathrm{a})$ ), while Rubicon knockdown decreased VP1 expression (Figure 4D(b)).

The effect of Rubicon on IAV replication was tested by measuring the production of three different forms of viral RNA (mRNA, cRNA and vRNA) in A549 cells. ${ }^{34}$ The results showed that Rubicon overexpression enhanced viral replication by 4-fold relative to the vector control (Figure 4E(a)). In contrast, Rubicon knockdown significantly inhibited viral replication (Figure 4E(b)).

VSV is extremely sensitive to the action of type-I IFNs. ${ }^{35}$ Therefore, we examined the effect of Rubicon on VSV replication in Huh7 cells. The results were similar to those observed for EV71 and IAV (Figure 4F(a and b)). Taken together, other viruses can induce Rubicon expression at both mRNA and protein levels.

\section{Rubicon affects the expression of IFNs and IFN downstream effectors}

The fact that Rubicon facilitates a broad range of virus infections prompted us to question whether Rubicon impairs the expression of IFNs, which are universal antiviral agents. ${ }^{36,37}$

First, we tested the effects of Rubicon overexpression and knockdown on cell viability. Our results demonstrated that neither condition significantly influenced the growth of Huh7 cells (data not shown). Next, we explored whether Rubicon affects IFN expression by using promoter-luciferase reporter assays. Rubicon expression inhibited Sendai virus (SeV)induced IFN- $\beta$ and IFN- $\lambda 1$ promoter activity (Figure $5 a$ ). 

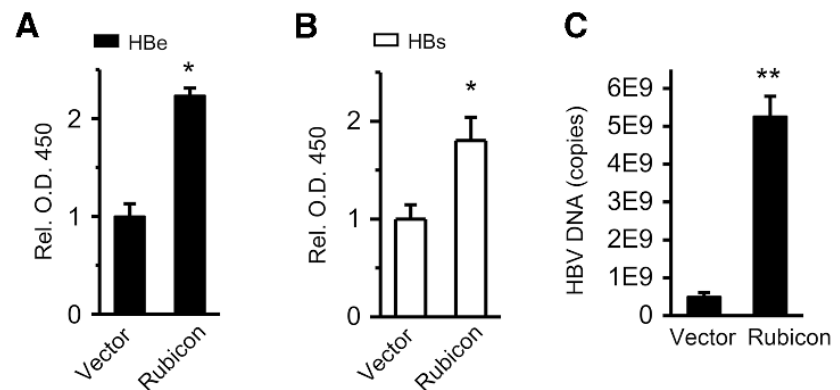

D

E

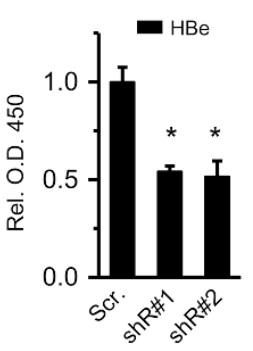

I a

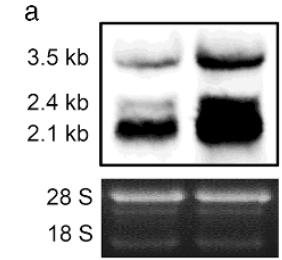

Ctrl Rubicon

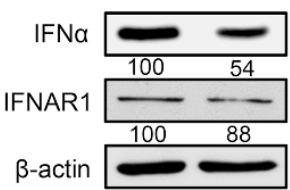

F

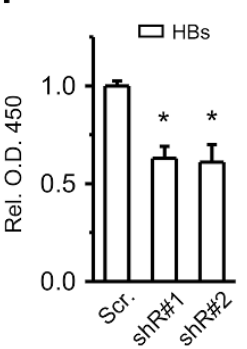

G

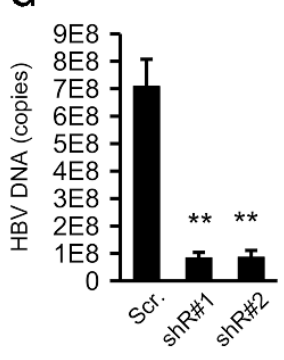

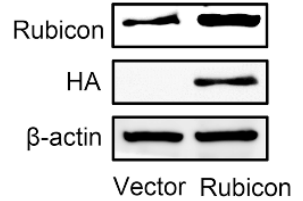

H Huh7

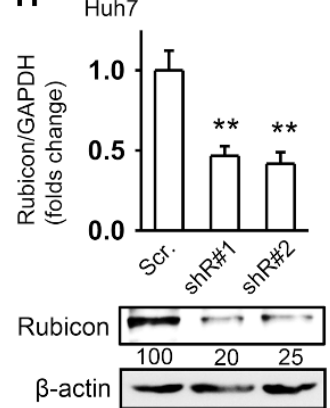

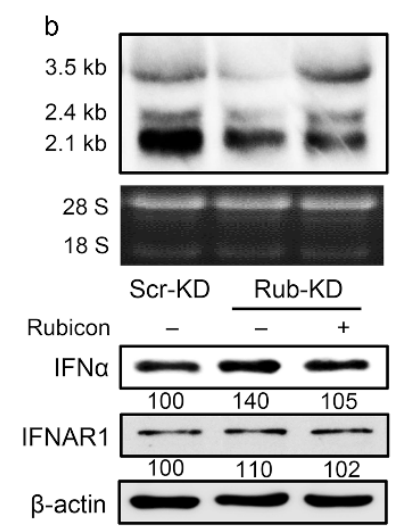

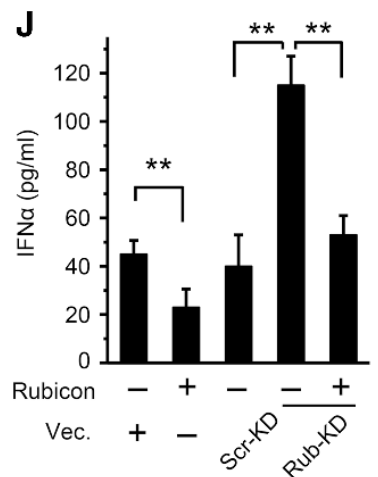

Figure 3 Rubicon increases virus replication. Huh7 cells were co-transfected with pHBV1.3D and plasmid expressing Rubicon (A-C) or Rubicon shRNA (shR) (E-G). At 48 h post-transfection, the supernatants were collected and assayed for HBeAg and HBsAg by ELISA, and the production of HBV DNA was analyzed by qPCR. An empty vector or an irrelevant shRNA was used as a control. (D) Huh7 cells were transfected with Rubicon expression plasmid or vector, and overexpression of Rubicon was detected by western blot analysis with the indicated antibodies; (H) Huh7 cells were transfected Rubicon shRNA (shR\#1 and shR\#2) or control shRNA (Scr). Forty-eight hours later, the cells were harvested for qRT-PCR after RNA isolation and western blotting. (I) Huh7 cells were co-transfected with pHBV1.3D and Rubicon expression plasmid or vector a), and Huh7 cells with stable Rubicon knockdown by shRNA or with scrambled shRNA were transfected with pHBV1.3D (b). After $48 \mathrm{~h}$, the production of HBV RNAs was measured by northern blotting. Simultaneously, the cells were harvested, the supernatants were collected and the protein was concentrated by ultra-filtration for western blot analysis. (J) Simultaneously, the IFN $\alpha$ levels in the cell culture supernatants were measured by ELISA. All experiments were repeated at least three times with consistent results. The data represent the mean \pm s.d., $n=3$. Bar graphs represent the mean \pm s.d., $n=3 \quad(* * P<0.01$; $\left.{ }^{*} P<0.05\right)$. HBV, hepatitis B virus; qRT-PCR, quantitative reverse transcriptase-PCR; shRNA, short hairpin RNA.

IFNs do not inhibit virus replication directly. Instead, IFN-stimulated genes (ISGs), including direct antiviral effectors such as 2',5'-oligoadenylate synthetase (OAS), myxovirus resistance A protein $(\mathrm{MxA})$, and protein kinase $\mathrm{R}(\mathrm{PKR})$, trigger antiviral effects. We assessed whether Rubicon could induce the production of endogenous IFNs and ISGs in Huh7 cells. The qRT-PCR analysis revealed that Rubicon suppressed virus-induced IFN- $\alpha$, IFN- $\beta$, and IFN- $\lambda 1$ mRNA levels (Figure 5b, upper panel). Western blot analysis revealed that Rubicon overexpression suppressed MxA, PKR, and OAS protein levels (Figure 5b, lower panel). Rubicon knockdown by specific shRNA consistently enhanced IFN (IFN- $\alpha$, IFN- $\beta$ and IFN- $\lambda 1$ ) mRNA levels (Figure $5 c$, upper panel) and ISG (MxA, PKR and OAS) protein levels (Figure 5c, lower panel).

As a hallmark of IFN production, IRF3/7 translocation play a very important role in IFN expression. We then examined the effect of Rubicon on the translocation of IRF3/7 from the cytosol to the nucleus. Western blot analysis revealed that IRF3/7 protein levels were increased in the cytosol and decreased in the nucleus after Rubicon overexpression in Huh7 cells induced by SeV (Figure 5d). Consistently, Rubicon knockdown enhanced SeV-induced translocation of IRF3/7 to 
A

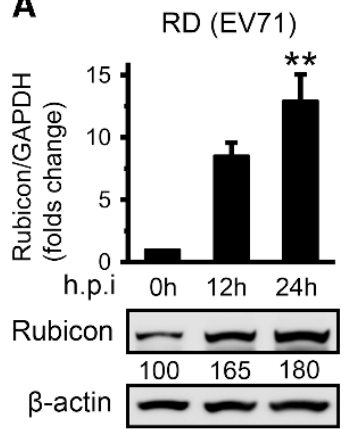

D a

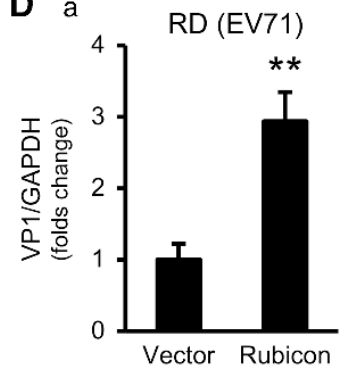

E a

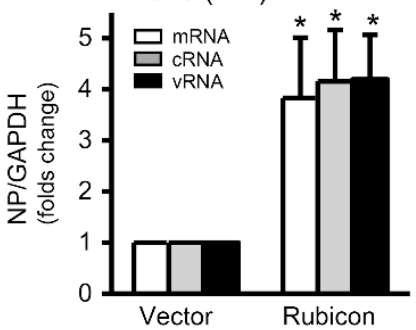

B

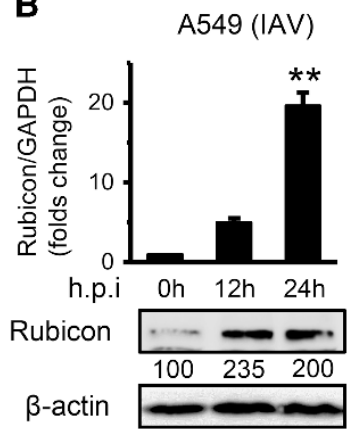

b

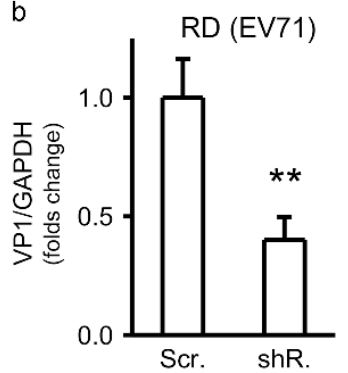

b

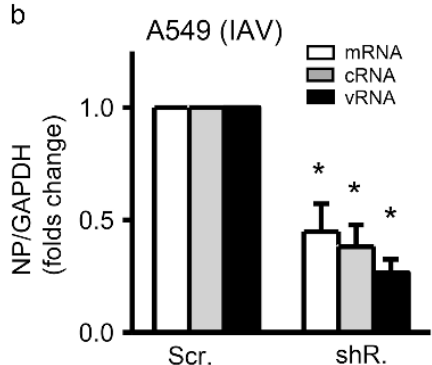

C

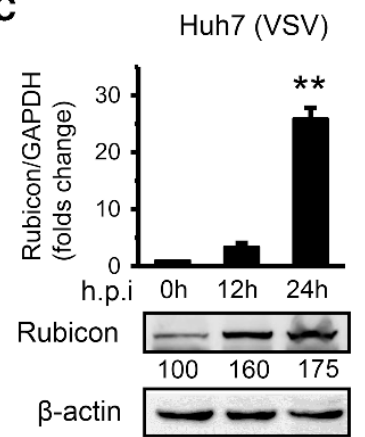

F a
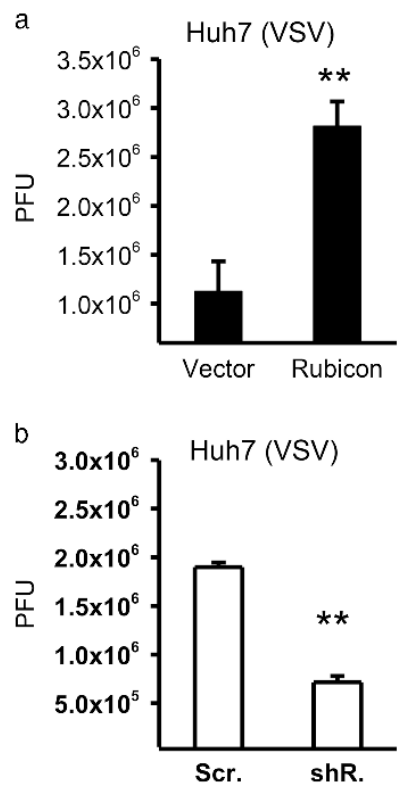

Figure 4 EV71, IAV and VSV induce Rubicon expression, and Rubicon enhances viral replication and production. (A-C) mRNA and protein levels were detected by qRT-PCR or western blotting in RD/A549/Huh7 cells with or without EV71/IAV/VSV infection. (D) RD cells were transfected with plasmid expressing Rubicon or vector for overexpression (a), or infected with lentivirus-shR or lentivirus-Scr for knockdown (b), and then infected with EV71 $(\mathrm{MOI}=1)$ for $12 \mathrm{~h}$ before harvesting the cells. After harvesting, the mRNA levels of the VP1 gene were analyzed by qRT-PCR. (E) A549 cells were transfected with plasmid expressing Rubicon or overexpression vector (a), or infected with lentivirus-shR or lentivirus-Scr for knockdown $(\mathbf{b})$, and then infected with IAV $(\mathrm{MOI}=1)$ for $8 \mathrm{~h}$ before cell harvest. After harvest, the mRNA levels of the IAV NP gene were analyzed by qRT-PCR. (F) Huh7 cells were transfected with plasmid expressing Rubicon or vector for overexpression (a), or infected with lentivirus-shR or lentivirus-Scr for knockdown (b), and then infected with VSV for 24 h. The culture medium was then harvested, and the viral loads were determined by plaque assay on Vero cells. All experiments were repeated at least three times with consistent results. The data represent the mean \pm s.d., $n=3$. Bar graphs represent the mean \pm s.d., $n=3$ (** $P<0.01$; $\left.{ }^{*} P<0.05\right)$. EV71, enterovirus 71; IAV, influenza A virus; qRT-PCR, quantitative reverse transcriptase-PCR; VSV, vesicular stomatitis virus.

the nucleus (Figure 5e). Taken together, these results indicate that Rubicon down-regulates IFNs and ISGs.

\section{NEMO is a Rubicon-associated protein}

Our observations strongly suggested that Rubicon plays a role in IFN signaling. We next examined the crosstalk between Rubicon and the IFN pathway. We overexpressed Rubicon, IFN pathway factors, and the IFN- $\beta$ promoter in Rubicon knockdown cells or Scramble knockdown cells to screen for the target of Rubicon. Luciferase activity ratios of GFP and IFNbeta promoter co-transfected in Rub-KD cells and Scr-KD cells were set as a control. These data showed that among those factors, NEMO and MAVS (also known as VISA, IPS-1, IPS1, and CARDIF) might be involved in the Rubicon-mediated IFN pathway (Figure 6a). We conducted co-immunoprecipitation (Co-IP) to detect the interaction between Rubicon and NEMO. Immune blots indicated that Rubicon interacts with NEMO but not with MAVS (Figure 6b). For further evidence, we constructed two plasmids containing GFP-tagged Rubicon and DsRed-tagged NEMO, respectively. Overexpression in HepG2.2.15 cells revealed co-localization of the two proteins (Figure 6c).

To determine which domain of Rubicon interacts with NEMO, we constructed four plasmids, each containing a different fragment. The first fragment (1-203) was the RUN domain of Rubicon. The other fragments (204-504, 1-504, and 

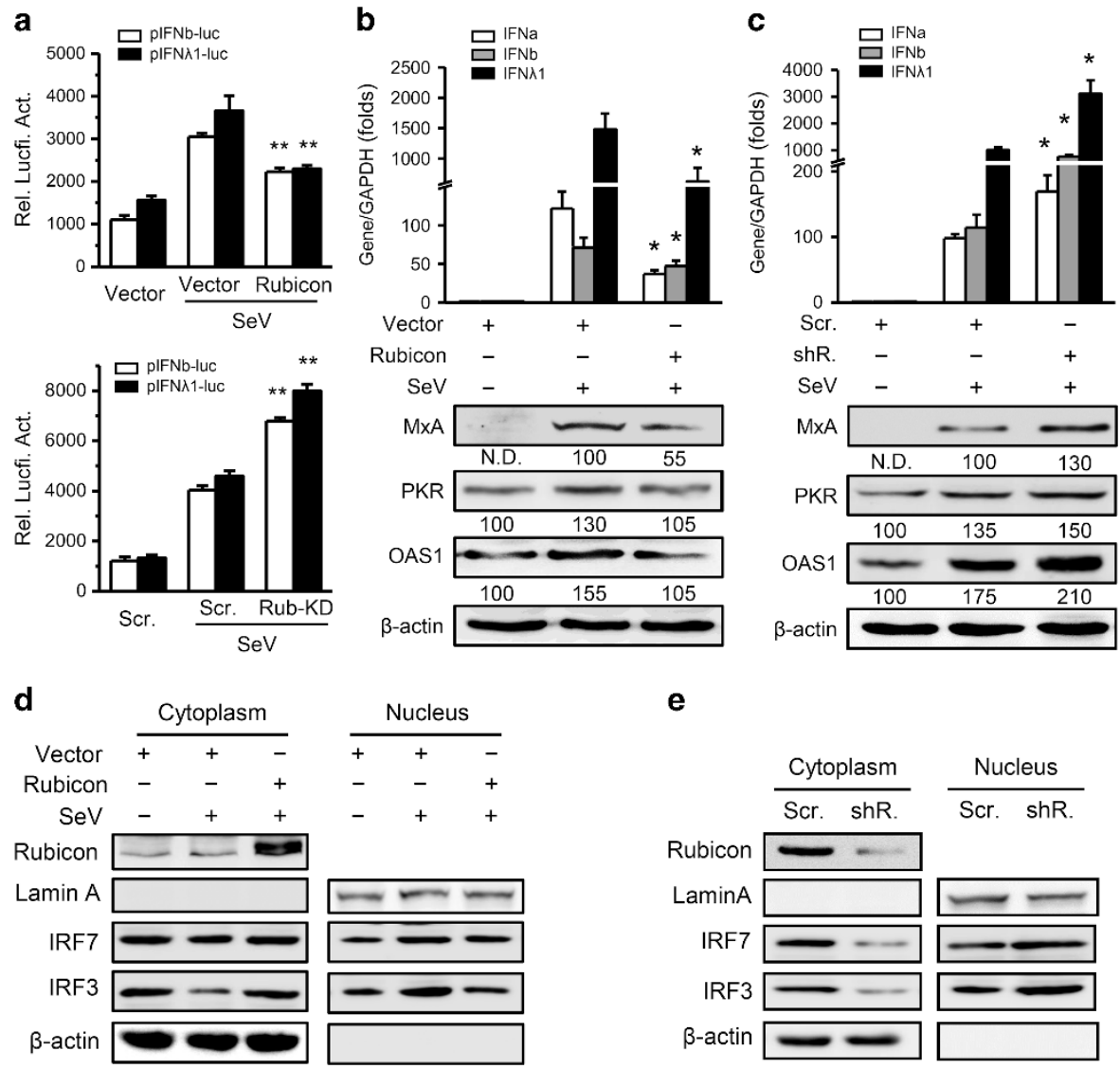

Figure 5 Rubicon downregulates IFN pathways. (a) Huh7 cells were co-transfected with luciferase reporter plasmids containing the IFN- $\beta$ or IFN- $\lambda 1$ promoter (pIFN- $\beta$-luc or pIFN- $\lambda 1$-luc) and plasmid expressing Rubicon or vector (up panel). Stable knockdown cell lines in Huh7 (Scr or Rub-KD) by lentivirus were transfected with luciferase reporter plasmids containing the IFN- $\beta$ or IFN- $\lambda 1$ promoter (pIFN- $\beta$-luc or pIFN- $\lambda 1-$ luc) or vector (down panel). The cell culture medium was refreshed with serum-free medium, and SeV (MOI $=1)$ was then added to induce Rubicon for $12 \mathrm{~h}$ before collection. Luciferase activities were measured $48 \mathrm{~h}$ post-transfection. (b) Huh7 cells were transfected with plasmid expressing Rubicon or vector for expression and harvested $48 \mathrm{~h}$ after transfection. Then, IFN (IFN- $\alpha$, IFN- $\beta$, IFN- $\lambda 1$ ) mRNAs and ISG (MxA, PKR and OAS) proteins were analyzed by qRT-PCR or western blotting with or without SeV for $12 \mathrm{~h}$ before harvest, and (d) cytosolic and nuclear extracts were subjected to western blot analysis after SeV induction for $3 \mathrm{~h}$. LaminA and $\beta$-actin were used as markers for the nuclear and cytosolic fractions, respectively. $\mathbf{c}$ and e are the same except for the use of stable Rubicon knockdown Huh7 cells (Scr or shR) for depletion by lentivirus. All experiments were repeated at least three times with consistent results. The data represent the mean \pm s.d., $n=3$. Bar graphs represent the mean \pm s.d., $n=3\left({ }^{*} P<0.01 ;{ }^{*} P<0.05\right)$. IFN- $\alpha$, interferon- $\alpha$; qRT-PCR, quantitative reverse transcriptase-PCR.

505-972) contained several other domains (Figure 6d). Co-IP analysis showed that the interaction with NEMO depended on the RUN domain (fragments 1-203 and 1-504; Figure 6e).

Next, we examined the endogenous interaction between Rubicon and NEMO in Huh7 cells. Endogenous Co-IP revealed that Rubicon interacts somewhat with NEMO before $\mathrm{SeV}$ infection and that the interaction becomes much stronger following $\mathrm{SeV}$ infection (Figure 6f). Taken together, these results demonstrate that Rubicon interacts with NEMO via the RUN domain upon virus infection.

\section{Rubicon inhibits the ubiquitination of NEMO and the} activation of downstream factors

Because the ubiquitination of NEMO is necessary for the activation of IFN signaling, we asked whether Rubicon could affect the ubiquitination of NEMO and downstream events.
We co-transfected Huh7 cells with HA-tagged Rubicon, the truncated constructs or the control vector along with flagtagged NEMO and myc-tagged Ubb. Co-IP showed that the overexpression of Rubicon or the RUN domain significantly suppressed the ubiquitination of NEMO (Figure 7a). Consistently, the knockdown of Rubicon in Huh7 cells showed a higher ubiquitination of NEMO (Figure $7 \mathrm{~b}$ ). It has been reported that Lys 285 and Lys309 are the key sites of NEMO in downstream IFN signaling. Therefore, we constructed two mutants (K285A and K309A) to test whether ubiquitination was blocked. Myc-tagged Ubb, wild-type NEMO or the mutants were co-transfected along with shRub or scramble shRNA into 293T cells. The results showed that the knockdown of Rubicon enhanced the ubiquitination of wild-type NEMO, while few effects on the mutants were observed (Figure 7c). 

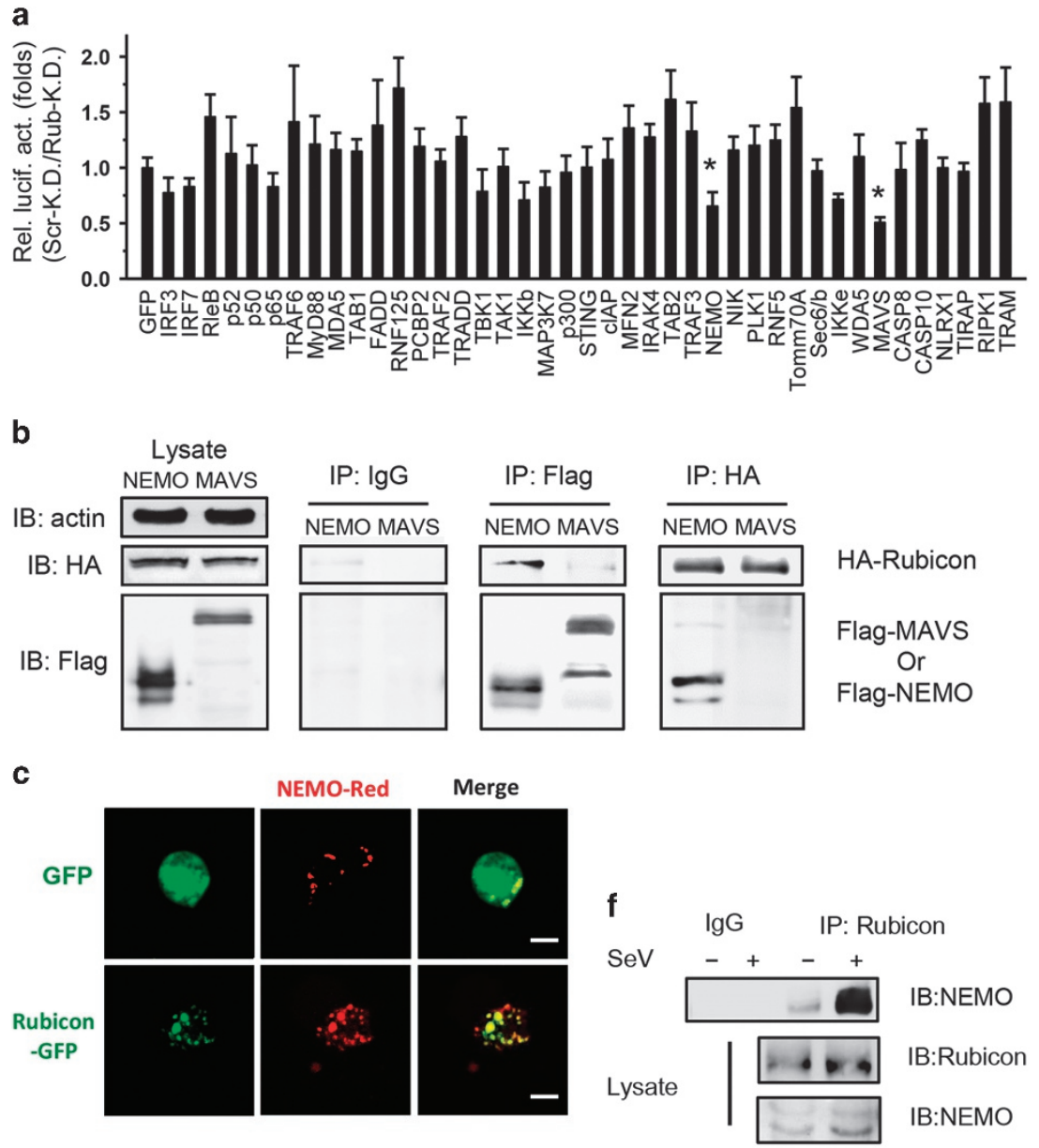

d

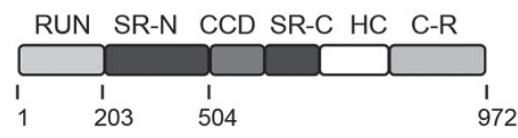

e

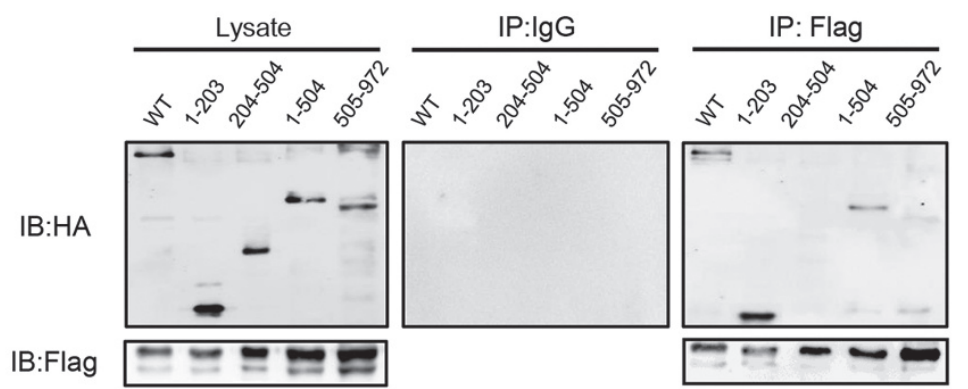

Figure 6 Rubicon interacts with NEMO. (a) Huh7 cells with stable Rubicon knockdown by shRNA or with scrambled shRNA were co-transfected with IFN-related factors and pIFN- $\beta$-luc. The luciferase activities were measured at $48 \mathrm{~h}$ post transfection after SeV induction for $12 \mathrm{~h}$. (b) HEK293T cells were transfected with MAVS or NEMO with the indicated tags along with Rubicon. At $48 \mathrm{~h}$ posttransfection, Co-IP and immunoblot analysis were performed with the indicated antibodies. The expression levels of transfected Rubicon, MAVS and NEMO were detected by western blotting. (c) HepG2.2.15 cells were co-transfected with NEMO-red and Rubicon-GFP or GFP. The cells were photographed with a confocal microscope $36 \mathrm{~h}$ after transfection. (d) Summary of wild-type and mutant Rubicon. (e) HEK293T cells were transfected with NEMO along with wild-type or mutant Rubicon with the indicated tags. At $48 \mathrm{~h}$ post transfection, Co-IP and immunoblot analyses were performed with the indicated antibodies. The expression levels of transfected NEMO and wild-type or mutant Rubicon were detected by western blotting. (f) Huh7 cells were stimulated with SeV (MOI=1) for $12 \mathrm{~h}$. Co-IP and immunoblot analyses were performed with the indicated antibodies. The expression of Rubicon and NEMO were then detected by western blotting. All experiments were repeated at least three times with consistent results. The data represent the mean \pm s.d., $n=3$. Bar graphs represent the mean \pm s.d., $n=3\left({ }^{*} P<0.01 ;{ }^{*} P<0.05\right)$. Co-IP, co-immunoprotection; IFN, interferon; shRNA, short hairpin RNA. 
a

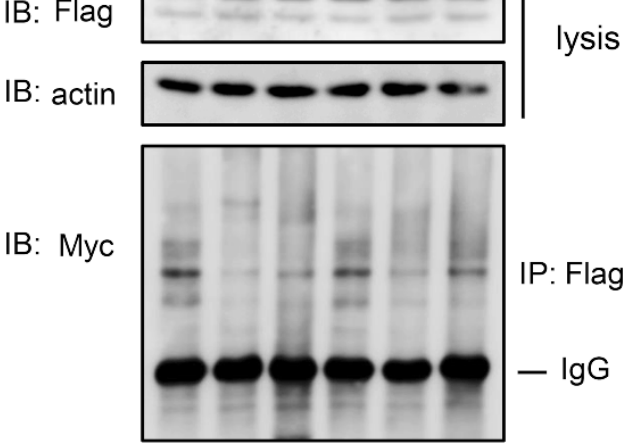

C

$\begin{array}{rrrrrrrrrr}\text { NEMO wt } & + & + & - & - & - & - & - & - \\ \text { K285A } & - & - & - & + & + & + & - & - & - \\ \text { K309A } & - & - & - & - & - & - & + & + & + \\ \text { Scr } & + & - & - & + & - & - & + & - & - \\ \text { ShR\#1 } & - & + & - & - & - & - & + & - \\ \text { ShR\#2 } & - & - & + & - & + & - & - & +\end{array}$

IB: Flag $2=0=2$

IB: actin

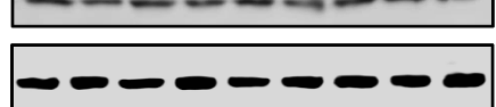

lysis

IB: Myc

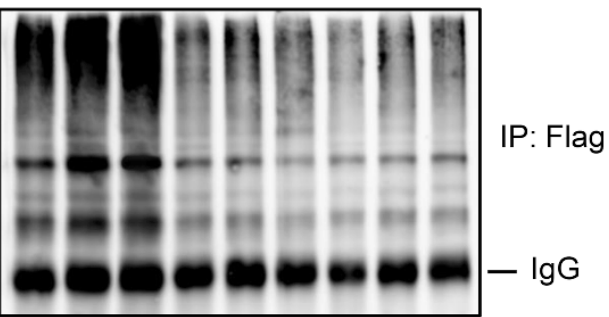

e

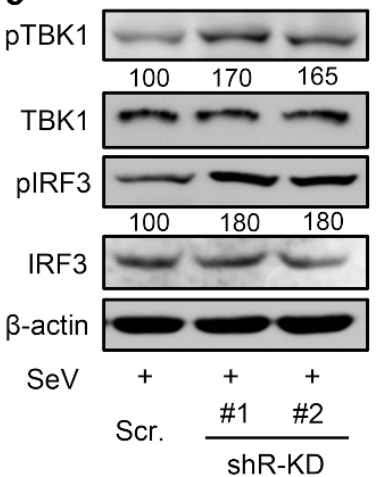

f pTBK1

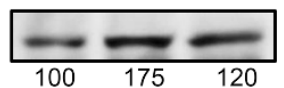

TBK1

pIRF3
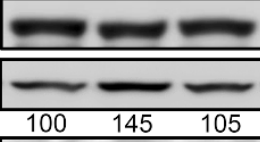

IRF3

$\beta$-actin

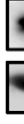

HBV1.3

Vector

Rubicon b

IB:

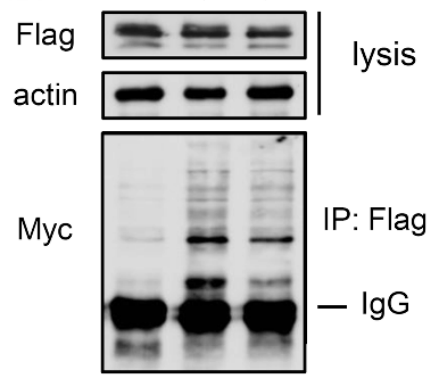

d

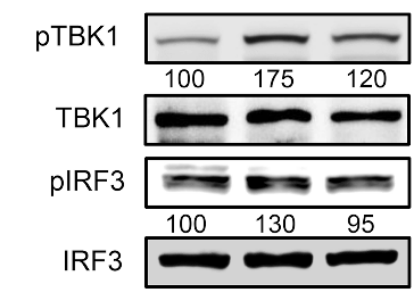

$\beta$-tubulin

$\mathrm{SeV}$

Vector

Rubicon

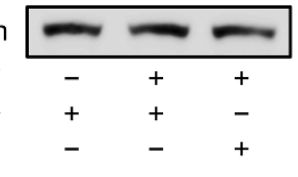

g

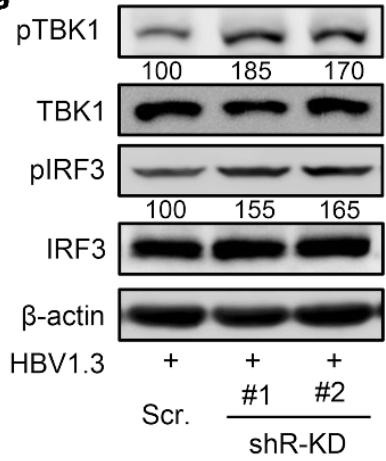

Figure 7 The interaction influenced ubiquitination of NEMO and downstream phosphorylation. (a) Huh7 cells were co-transfected with Flag-NEMO, HA-Rubicon or the mutants, and myc-Ubb. After $48 \mathrm{~h}$, the cells were harvested for IP and immunoblot analysis with the indicated antibodies. (b) Stable Rub-KD cells were co-transfected with Flag-NEMO and myc-Ubb. After $48 \mathrm{~h}$, the cells were harvested for immunoprecipitation (IP) and immunoblot analysis with the indicated antibodies. (c) 293T cells were co-transfected with the indicated plasmids and myc-Ubb. After $48 \mathrm{~h}$, the cells were collected for IP and immunoblot analysis with the indicated antibodies. (d) Huh7 cells transfected with plasmid expressing Rubicon or vector, (e) Stable Rub-KD cell lines or Scr. cells were analyzed by western blotting with the indicated antibodies at $48 \mathrm{~h}$ post transfection with or without $\mathrm{SeV}(\mathrm{MOI}=1)$ induced for $3 \mathrm{~h}$. (f) Huh7 cells transfected with the plasmid expressing Rubicon and HBV1.3 or vector and HBV1.3, and (g) Stable Rub-KD cell lines or Scr. Cells were transfected with HBV1.3. After $48 \mathrm{~h}$, the cells were collected and analyzed by western blotting with the indicated antibodies. All experiments were repeated at least three times with consistent results. HBV, hepatitis B virus. 
Next, we evaluated the phosphorylation of downstream factors (TBK1 and IRF3) by western blotting using phosphor-specific antibodies. Rubicon overexpression inhibited the phosphorylation of TBK1 and IRF3 (Figure 7d), while Rubicon knockdown had the opposite effect during $\mathrm{SeV}$ infection (Figure 7e). Similar results were observed in HBV1.3-transfected Huh7 cells (Figures $7 \mathrm{f}$ and g).

Overall, the association between Rubicon and NEMO inhibits the ubiquitination of NEMO and the phosphorylation of TBK1 and IRF3, which are necessary for IFN signal transduction.

\section{DISCUSSION}

In this study, we found that the host factor Rubicon can be induced during virus infection and facilitates virus replication. The induced Rubicon interacts with NEMO, an important factor in the IFN pathway. This interaction inhibits NEMO ubiquitination, TBK1 phosphorylation and IRF3/7 translocation from the cytoplasm to the nucleus, as well as, eventually, IFN production, which is the key factor in the host anti-virus effect (Figure 8).

In a previous report of Rubicon expression induced by $\mathrm{HCV}$ infection, ${ }^{29} \mathrm{HCV}$-infected Huh7.5 cells had elevated Rubicon expression levels, which delayed autophagosome maturation and enhanced HCV replication. In our study, patients with CHB had significantly increased Rubicon levels in liver tissues and PBMCs compared with healthy individuals (Figure 1). $\mathrm{HBV}$ induced Rubicon expression in freshly isolated PBMCs, and the induction could be blocked by HBV immunoglobulin. HBV also induced Rubicon expression at the transcriptional and protein levels in vitro (Figure 2). These results indicate that further study of the mechanism of Rubicon induction by HBV is warranted.

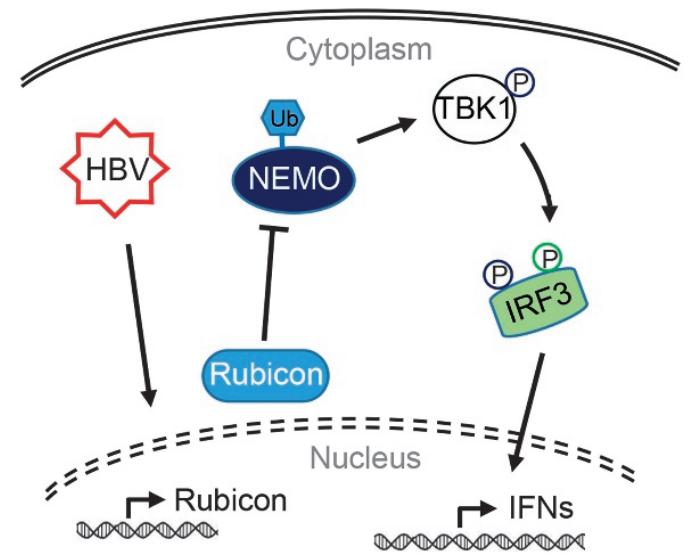

Figure 8 Summary of inducible Rubicon facilitation of HBV replication via the suppression of interferon signaling. Solid arrows represent signaling pathways identified in this study. Viruses (HBV, VSV, IAV and EV71) induce Rubicon expression. Then, Rubicon binds to NEMO to suppress ubiquitination while reducing IFN production, and enhances viral replication and production. EV71, enterovirus 71; HBV, hepatitis B virus; IAV, influenza A virus; VSV, vesicular stomatitis virus.
Autophagy enhances HBV production, ${ }^{38-40}$ but we found that Rubicon, an inhibitor of autophagy, could also promote HBV replication and production (Figure 3). Moreover, the changes in IFN $\alpha$ and IFNAR1 indicated that Rubiconenhanced HBV replication may not be due to autophagy. Furthermore, EV71, IAV and VSV-induced Rubicon expression and displayed increased replication facilitated by Rubicon (Figure 4). These results indicate that Rubicon influences HBV replication via routes other than autophagy.

Rubicon assumes different roles under a variety of physiological and pathological conditions. ${ }^{15,29,41}$ In one study, Rubicon changed its partner from the Beclin-1 complex to CARD9 in a Dectin-1-stimulation-dependent or RIG-I-stimulationdependent, competitive manner, thereby disassembling the CARD9, BCL10 and MALT1 signaling complex and suppressing NF- $\mathrm{KB}$ activation, ultimately stopping PRR-induced inflammatory cytokine production. ${ }^{15,42}$

IFN- $\alpha$ is widely used to treat $\mathrm{HBV}$ infection because HBV is strongly inhibited by IFNs in some patients, and IFNs are important defenders of the innate immune system. ${ }^{43}$ However, IFNs are not the direct agents of the antiviral response. ISGs (most importantly, PKR, OAS, and MxA) are the actual antiviral effectors. ${ }^{44,45}$ It has been well demonstrated that the induction of type-I IFNs is required for activation of the IRF3/7 and NF- $\mathrm{\kappa B}$ pathways. ${ }^{46,47}$ IRF3, a constitutively expressed, phosphorylation-dependent regulatory factor, is activated by virus-induced phosphorylation, which leads to homodimerization, heterodimerization and nuclear translocation as well as its association with the co-activator $\mathrm{CBP} / \mathrm{p} 300 .{ }^{48,49}$ Similar to IRF3, IRF7 is well-known as an important regulator of virusinduced IFN- $\alpha$ expression that depends on phosphorylation and nuclear translocation. ${ }^{47}$ The requirement for NF- $\mathrm{\kappa B}$ in IFN- $\beta$ regulation has been recognized for decades. Rubicon has been reported to suppress NF- $\mathrm{KB}$ signaling upon microbe infection, so we mainly focused on IRF3/7 induction of type-I IFNs. We showed that Rubicon was able to suppress the expression of IFN-induced PKR, OAS, and MxA (Figure 5). To identify the role of Rubicon in the IFN signaling pathway during HBV infection, we showed that the IKK family factor NEMO was the target to transport the signal (Figure 6). NEMO is a key factor in the phosphorylation of IRF3 and the production of type-I IFNs. The absence of NEMO can therefore lead to uncontrolled virus replication. ${ }^{12}$ The interaction of ubiquitin oligomers with NEMO activates IKK $\alpha$ and IKK $\beta$ by phosphorylation, leading to IкB- $\alpha$ phosphorylation and subsequent NF- $\mathrm{KB}$ activation. ${ }^{50,51}$ NEMO can bind to TANK, TBK1 and IKKe by forming a complex to phosphorylate Ser172 of TBK1 and IRF3, which leads to IFN production. Our results suggest that Rubicon interacts with NEMO via the RUN domain to inhibit NEMO ubiquitination, TBK1 and IRF3 phosphorylation, and eventually IFN production.

It is important for organisms to maintain balance, including of the immune system. Depending on the environmental stimuli, Rubicon is involved in three different ancient innate immune machineries: autophagy, phagocytosis, and PRRs. For efficiency, Rubicon differentially targets signaling complexes, 
depending on environmental stimuli, and might function to coordinate various immune responses against viral infection. We showed that Rubicon can be induced by viral infection and suppresses IFN signal by targeting NEMO, inhibiting ubiquitination though the RUN domain. Our results reveal a previously unrecognized function of Rubicon as an important negative regulator of the innate immune response.

\section{CONFLICT OF INTEREST}

The authors declare no conflict of interest.

\section{ACKNOWLEDGEMENTS}

We are grateful for the supporting of special funding for the $\mathrm{PhD}$ students of Wuhan University short-term study abroad, and we thank Tielong Chen in Zhongnan Hospital of Wuhan University for kindly providing HBV patient samples in this study. This work was supported by research grants from the Major State Basic Research Development Program of China (2013CB911102) and the National Natural Science Foundation of China (81461130019, 81271821 and 31570870). The funding agencies had no role in the study design, data collection or analysis, decision to publish or preparation of the manuscript.

1 Vilcek J. Fifty years of interferon research: aiming at a moving target. Immunity 2006; 25: 343-348.

2 Pestka S, Krause CD, Walter MR. Interferons, interferon-like cytokines, and their receptors. Immunol Rev 2004; 202: 8-32.

3 Borden EC, Sen GC, Uze G, Silverman RH, Ransohoff RM, Foster GR et al. Interferons at age 50: past, current and future impact on biomedicine. Nat Rev Drug Discov 2007; 6: 975-990.

4 Christensen $\mathrm{MH}$, Paludan SR. Viral evasion of DNA-stimulated innate immune responses. Cell Mol Immunol 2016; 14: 4-13.

5 Du Q, Xie J, Kim HJ, Ma X. Type I interferon: the mediator of bacterial infection-induced necroptosis. Cell Mol Immunol 2013; 10: 4-6.

6 Rothwarf DM, Zandi E, Natoli G, Karin M. IKK-gamma is an essential regulatory subunit of the IkappaB kinase complex. Nature 1998; 395: 297-300.

7 Yamaoka S, Courtois G, Bessia C, Whiteside ST, Weil R, Agou F et al. Complementation cloning of NEMO, a component of the IkappaB kinase complex essential for NF-kappaB activation. Cell 1998; 93: 1231-1240.

8 Clark K, Nanda S, Cohen P. Molecular control of the NEMO family of ubiquitin-binding proteins. Nat Rev Mol Cell Biol 2013; 14: 673-685.

9 Clark K, Takeuchi O, Akira S, Cohen P. The TRAF-associated protein TANK facilitates cross-talk within the I kappa B kinase family during Toll-like receptor signaling. Proc Natl Acad Sci USA 2011; 108: 17093-17098.

10 Fitzgerald KA, McWhirter SM, Faia KL, Rowe DC, Latz E, Golenbock DT et al. IKKepsilon and TBK1 are essential components of the IRF3 signaling pathway. Nat Immunol 2003; 4: 491-496.

11 Sharma S, tenOever BR, Grandvaux N, Zhou GP, Lin R, Hiscott J. Triggering the interferon antiviral response through an IKK-related pathway. Science 2003; 300: 1148-1151.

12 Zhao T, Yang L, Sun Q, Arguello M, Ballard DW, Hiscott J et al. The NEMO adaptor bridges the nuclear factor-kappaB and interferon regulatory factor signaling pathways. Nat Immunol 2007; 8: 592-600.

13 Matsunaga K, Noda T, Yoshimori T. Binding Rubicon to cross the Rubicon. Autophagy 2009; 5: 876-877.

14 Zhong Y, Wang QJ, Li XT, Yan Y, Backer JM, Chait BT et al. Distinct regulation of autophagic activity by Atg14L and Rubicon associated with Beclin 1-phosphatidylinositol-3-kinase complex. Nat Cell Biol 2009; 11: 468-U262.

15 Yang CS, Rodgers M, Min CK, Lee JS, Kingeter L, Lee JY et al. The autophagy regulator Rubicon is a feedback inhibitor of CARD9- mediated host innate immunity. Cell Host Microbe 2012; 11: 277-289.

16 Sato S, Li K, Kameyama T, Hayashi T, Ishida Y, Murakami S et al. The RNA Sensor RIG-I Dually Functions as an Innate Sensor and Direct Antiviral Factor for Hepatitis B Virus. Immunity 2015; 42: 123-132.

17 Luangsay S, Gruffaz M, Isorce N, Testoni B, Michelet M, Faure-Dupuy $\mathrm{S}$ et al. Early inhibition of hepatocyte innate responses by hepatitis B virus. J Hepatol 2015; 63: 1314-1322.

18 Liu S, Peng N, Xie J, Hao Q, Zhang M, Zhang Y et al. Human hepatitis $B$ virus surface and e antigens inhibit major vault protein signaling in interferon induction pathways. J Hepatol 2015; 62: 1015-1023.

19 Choi HJ, Dinarello CA, Shapiro L. Interleukin-18 inhibits human immunodeficiency virus type 1 production in peripheral blood mononuclear cells. J Infect Dis 2001; 184: 560-568.

20 Werner M, Driftmann S, Kleinehr K, Kaiser GM, Mathe Z, Treckmann JW et al. All-in-one: advanced preparation of human parenchymal and non-parenchymal liver cells. PLOS ONE 2015; 10: e0138655.

21 Yue X, Wang H, Zhao F, Liu S, Wu J, Ren W et al. Hepatitis B virusinduced calreticulin protein is involved in IFN resistance. $J$ Immunol 2012; 189: 279-286.

22 Li Y, Xie J, Xu X, Liu L, Wan Y, Liu Y et al. Inducible interleukin 32. IL-32) exerts extensive antiviral function via selective stimulation of interferon lambda1. IFN-lambda1. J Biol Chem 2013; 288: 20927-20941.

23 Liu D, Cui L, Wang Y, Yang GF, He J, Hao RD et al. Hepatitis B e Antigen and Its Precursors Promote the Progress of Hepatocellular Carcinoma by Interacting With NUMB and Decreasing p53 Activity. Hepatology 2016; 64: 390-404.

24 Sun QM, Zhang J, Fan WL, Wong KN, Ding XJ, Chen S et al. The RUN domain of rubicon is important for hVps34 binding, lipid kinase inhibition, and autophagy suppression. J Biol Chem 2011; 286: 185-191.

25 Yu ZB, Fotouhi-Ardakani N, Wu LT, Maoui M, Wang SL, Banville D et al. PTEN associates with the vault particles in HeLa cells. J Biol Chem 2002; 277: 40247-40252.

26 Han T, Wan Y, Wang J, Zhao P, Yuan Y, Wang L et al. Set7 facilitates hepatitis $C$ virus replication via enzymatic activity-dependent attenuation of the IFN-related pathway. Journal of immunology 2015; 194: 2757-2768.

27 Belloni L, Allweiss L, Guerrieri F, Pediconi N, Volz T, Pollicino T et al. IFN-alpha inhibits HBV transcription and replication in cell culture and in humanized mice by targeting the epigenetic regulation of the nuclear cccDNA minichromosome. J Clin Invest 2012; 122: 529-537.

28 Shu HB, Takeuchi M, Goeddel DV. The tumor necrosis factor receptor 2 signal transducers TRAF2 and C-IAP1 are components of the tumor necrosis factor receptor 1 signaling complex. Proc Natl Acad Sci USA 1996; 93: 13973-13978.

29 Wang L, Tian Y, Ou JH. HCV induces the expression of Rubicon and UVRAG to temporally regulate the maturation of autophagosomes and viral replication. PLoS Pathog 2015; 11: e1004764.

30 Tong S, Revill P. Overview of hepatitis B viral replication and genetic variability. J Hepatol 2016; 64: S4-16.

31 Zhao F, Xu G, Zhou Y, Wang L, Xie J, Ren S et al. MicroRNA-26b inhibits hepatitis $B$ virus transcription and replication by targeting the host factor CHORDC1 protein. J Biol Chem 2014; 289. 35029-35041.

32 Geng M, Xin X, Bi LQ, Zhou LT, Liu XH. Molecular mechanism of hepatitis $\mathrm{B}$ virus $\mathrm{X}$ protein function in hepatocarcinogenesis. World $\mathrm{J}$ Gastroenterol 2015; 21: 10732-10738.

33 Decorsiere A, Mueller H, van Breugel PC, Abdul F, Gerossier L, Beran RK et al. Hepatitis $B$ virus $X$ protein identifies the Smc5/6 complex as a host restriction factor. Nature 2016; 531: 386-389.

34 Liu S, Hao Q, Peng NF, Yue X, Wang Y, Chen YN et al. Major vault protein: A virus-induced host factor against viral replication through the induction of type-I interferon. Hepatology 2012; 56: 57-66.

35 Kramer MJ, Dennin R, Kramer C, Jones G, Connell E, Rolon N et al. Cell and virus sensitivity studies with recombinant human alpha interferons. J Interferon Res 1983; 3: 425-435. 
36 Yang C, Zhao X, Sun D, Yang L, Chong C, Pan Y et al. Interferon alpha. IFNalpha)-induced TRIM22 interrupts HCV replication by ubiquitinating NS5A. Cell Mol Immunol 2016; 13: 94-102.

37 Wong MT, Chen SS. Emerging roles of interferon-stimulated genes in the innate immune response to hepatitis $\mathrm{C}$ virus infection. Cell $\mathrm{Mol}$ Immunol 2016; 13: 11-35.

38 Silva LM, Jung JU. Modulation of the autophagy pathway by human tumor viruses. Semin Cancer Biol 2013; 23: 323-328.

39 Tian Y, Sir D, Kuo CF, Ann DK, Ou JH. Autophagy required for hepatitis B virus replication in transgenic mice. Journal of virology 2011; 85: 13453-13456.

40 Li J, Liu Y, Wang Z, Liu K, Wang Y, Liu J et al. Subversion of cellular autophagy machinery by hepatitis B virus for viral envelopment. Journal of virology 2011; 85: 6319-6333.

41 Yang CS, Lee JS, Rodgers M, Min CK, Lee JY, Kim HJ et al. Autophagy protein Rubicon mediates phagocytic NADPH oxidase activation in response to microbial infection or TLR stimulation. Cell Host Microbe 2012; 11: 264-276.

42 Wang J, Wang Q, Han T, Li YK, Zhu SL, Ao F et al. Soluble interleukin-6 receptor is elevated during influenza $A$ virus infection and mediates the IL-6 and IL-32 inflammatory cytokine burst. Cell Mol Immunol 2015; 12: 633-644.

43 Ren S, Wang J, Chen TL, Li HY, Wan YS, Peng NF et al. Hepatitis B Virus Stimulated Fibronectin Facilitates Viral Maintenance and Replication through Two Distinct Mechanisms. PLoS One 2016; 11.
44 Clemens MJ, Elia A. The double-stranded RNA-dependent protein kinase PKR: structure and function. J Interferon Cytokine Res 1997; 17: 503-524.

45 Yakub I, Lillibridge KM, Moran A, Gonzalez OY, Belmont J, Gibbs RA et al. Single nucleotide polymorphisms in genes for 2'-5'-oligoadenylate synthetase and RNase L inpatients hospitalized with West Nile virus infection. J Infect Dis 2005; 192: 1741-1748.

46 Bonjardim CA, Ferreira PC, Kroon EG. Interferons: signaling, antiviral and viral evasion. Immunol Lett 2009; 122: 1-11.

47 Honda K, Takaoka A, Taniguchi T. Type I inteferon gene induction by the interferon regulatory factor family of transcription factors. Immunity 2006; 25: 349-360.

48 Lin R, Mamane Y, Hiscott J. Structural and functional analysis of interferon regulatory factor 3 : localization of the transactivation and autoinhibitory domains. Mol Cell Biol 1999; 19: 2465-2474.

49 Weaver BK, Kumar KP, Reich NC. Interferon regulatory factor 3 and CREB-binding protein/p300 are subunits of double-stranded RNAactivated transcription factor DRAF1. Mol Cell Biol 1998; 18: 1359-1368.

50 Rahighi S, Ikeda F, Kawasaki M, Akutsu M, Suzuki N, Kato R et al. Specific recognition of linear ubiquitin chains by NEMO is important for NF-kappa B activation. Cell 2009; 136: 1098-1109.

51 Lo YC, Lin SC, Rospigliosi CC, Conze DB, Wu CJ, Ashwell JD et al. Structural basis for recognition of diubiquitins by NEMO. Mol Cell 2009; 33: 602-615.

Supplementary Information for this article can be found on the Cellular \& Molecular Immunology website (http://www.nature.com/cmi) 